Aus dem Laboratorium der Königl. Frauenklinik in Dresden. Director: Geh. Med.-Rath Prof. Dr. Leopold.

\title{
Beitrag zur Tuberculose der weiblichen Adnexe.
}

Von

\section{Dr. Loiacono.}

Die Forschungen über die weibliche Genitaltuberculose haben zu verschiedenen Zeiten verschiedene Richtungen gehabt.

Einen Beweis dafür gab der internationale Gynäkologencongress in Rom, auf welchem die Hauptfrage erörtert wurde, ob bei Erauen eine primäre Genitaltuberculose vorkomme.

Während A. Martin, Veit, Pichevin, Pozzi, Spinelli und Gutierrez auf Grund eigener und anderer Untersuchungen erklärten, dass eine primäre Genitaltuberculose bei Frauen, wenn auch nur selten, beobachtet werde, nahm Amann an, dass die Entstehung einer primären Genitaltuberculose durch Ansteckung von aussen in hohem Grade problematisch sei. Nach seiner Meinung liesse sich über das von anderen berichtete Vorkommen der primären Genitaltuberculose streiten, weil der klinische und operative Befund unzulänglich sei.

Nur der auf Grundlage der Autopsie gemachte Befund und nur der Befund in solchen Fällen, in welchen zu einem bestimmten Zweck die in anderen Organen verborgenen Herde untersucht worden sind, könne uns in die Lage setzen, einen primären Genitaltuberculoseprocess als vorhanden anzusehen oder zu verneinen.

Auch Bertrix bestätigte auf dem genannten Congresse durch die Beschreibung einer von $\mathrm{ihm}$ ausgeführten Untersuchung die Ansichten Amann's. In diesem Falle handelte es sich um eine Patientin, welche wach einer Adnexoperation starb. Die Autopsie zeigte Tuberculose der peribronchialen Drüsen und tubereulöse Herde im Epiploon und in der Leber.

Bertrix nahm an, dass sich der tuberculöse Process von den peribronchialen Drüsen auf hämatogenem Wege in der Leber, im 
Epiploon und in den Adnexen verbreitet habe; ohne Autopsie würde man eine primäre Genitaltuberculose vermuthet haben.

Es giebt Fälle, in welchen die Adnexe von dem tuberculösen Process ergriffen sind und das umgebende Peritoneum unversehrt ist; sie verleiten zu der Annahme, dass der tuberculöse Process in den Adnexen seinen primären Ursprung hat. Nach Glimm kann man sich jedoch in dieser Vermuthung bei den einzelnen Fällen täuschen, denn wo keine Peritonitis ist, kann die Ansteckung der Tuben und der Ovarien ihren Weg vom Peritoneum aus genommen haben, ohne dass dieses von der Tuberculose ergriffen worden ist. Pichevin bebauptet in einem seiner neuesten Aufsätze in der "Sem. Gynaecologique", dass man sehr genaue Untersuchungen vornehmen müsse, bevor man, angesichts der grossen Häufigkeit der Lungentuberculose, "deren Existenz bei Frauen, welche Symptome der Genitaltuberculose aufweisen, verneint. Auf Grund einer solchen Behauptung, mit welcher die meisten übereinstimmen, zeigen die Fälle der primären Genitaltuberculose für das Einsetzen der Kritik grösstentheils diese schwache Seite. Maenaugton-Jones beschreibt einen Fall, bei welchem einer Patientin die Adnexe einer Seite in Folge eines tuberculösen Processes entfernt worden waren. Bei der Operation zeigte sich der übrige Genitalapparat vollkommen gesund. Die Patientin hatte in der Folgezeit vier Geburten, darunter eine Zwillingsgeburt. Nach der letzten wurde die Frau von neuem in Folge postpuerperaler Perimetritis operirt und die Adnexe der anderen Seite entfernt. Diese waren frei von Tuberculose. Nach Feststellung dieser Thatsachen behauptet der Verfasser, dass die zuerst entfernten Adnexe von einem primären Tuberculoseprocess ergriffen waren. Die Vertreter der gegentheiligen Ansicht behaupten in diesem Falle, dass ein kleiner Herd in den Lungen oder in anderen Organen den Process in den Adnexen hervorgerufen haben könne.

In den letzten Jahren hat man die besprochene Frage durch Experimente zu lösen versucht, aber auch hierbei sind keine übereinstimmenden Resultate erzielt worden.

Während die experimentellen Versuche von Jung und Bennecke zeigen, dass man eine primäre und ascendirende Genitaltuberculose bei Kaninchen erzeugen kann (denn unter 82 Kaninchen, die auf verschiedene Arten geimpft worden waren, wurde bei zwölf Thieren die ascendirende Tuberculose nachgewiesen), haben die Versuche Blau's entgegengesetzte Resultate erzielt. Er verwendete für seine 
Versuche Meerschweinchen und inficirte theils mit tuberculösem Gewebe von Meerschweinchen, deren Peritoneum mit menschlichen Tuberkelbacillen inficirt worden war, theils verwendete er dazu Reinculturen von menschlichen Tuberkelbacillen.

In einer Reihe von Versuchen wurde die inficirte Substanz in Thiere mit unverletzter Vagina, in einer anderen Reihe mit zuvor verletzter Vagina eingeführt. Ausserdem war eine Reihe von Thieren, die sich im Puerperium oder am Ende der Schwangerschaft befanden, auf dem Vaginalwege inficirt worden. Endlich in einer anderen Reibe von Versuchen hat man die Gebärmutter mittels Operation zur Sicht gebracht and direct inficirt. In dieser letzten Gruppe von Versuchen hatte der Forscher die Gebärmutter sowohl im Puerperalzustand, als auch im normalen Zustand inficirt, indem er Reinculturen von Tuberkelbacillen verwendete.

Man ersah hieraus, dass das Meerschweinchen, welches sich ausserordentlich empfindlich für die Tuberculose zeigt, für die Ansteckung in den Geschlechtstheilen nur in dem Puerperalzustand und nach örtlichen Verletzungen grosse Empfänglichkeit aufwies. Ein ascendirender Process war in keinem Falle nachweisbar.

Blau berichtet über 36 Fälle von Genitaltuberculose an der Chrobak'schen Klinik, von denen 33 operirt wurden. Von diesen waren bei 25 ausschliesslich die Adnexe, bei 8 die Adnexe und Gebärmutter, bei 3 nur die Gebärmutter erkrankt. Unter den Erkrankungen der Adnexe fanden sich sechsmal die Eierstöcke inficirt, auch das Peritoneum war in 24 Fällen mit erkrankt. Im Gegensatz dazu waren unter 109 Fällen, in welchen die Operation in Folge ron Peritonealtuberculose ausgeführt worden war, in 89 Fällen. die Adnexe makroskopisch nicht verändert.

Die Resultate der klinischen Beobachtungen führen den Forscher zu dem Schluss, dass in keinem Falle eine primäre tuberculöse Infection oder ein ascendirender Process nachweisbar war. Wie bei Meerschweinchen die einfache Einführung tuberculösen Gewebes von hoher Giftigkeit nur bei gewissen prädisponirenden Momenten (Puerperiumverletzungen) Tuberculose hervorruft, so beobachtet man auch bei Frauen, in Folge der Wirkung des das Leben der Tuberkelbacillen hemmenden Vaginalsecrets und in Folge des dicken Epitheliums auf der unteren Strecke des Genitalweges, welcher der Einführung der Bacillen nicht günstig ist, dass nur eine geringe Möglichkeit für eine Ansteckung vorhanden ist. Die Entstehung einer primären Genitaltuberculose würde also bei Frauen nur auf günstigem 
Boden (Puerperiumverletzungen) und bei hoher Virulenz der Bacillen denkbar sein.

Bei diesen verschiedenen Meinungen stimmen alle Forscher darin überein, dass eine genaue Feststellung für den Gynäkologen mit grossen Schwierigkeiten verknüpft ist. Für die Entwickelung der Genitaltuberculose scheint hauptsächlich das Alter von 20 bis 40 Jahren in Betracht zu kommen, das Sexualleben scheint sogar einen schon vorhandenen Tuberculoseprocess noch zu verschlimmern.

Die Genitaltuberculose kommt auch im jugendlichen Alter vor. So berichtet Hohlfefd über ein Kind von 3 Jahren und ein solches von 1 Jahr 7 Monaten mit Tuberculose in anderen Organen und bei der Autopsie mit Genitaltuberculose, ferner kam Bouilly durch seine Forschung über Ascites bei Kindern zu dem Ergebnisse, dass der Ascites seinen Ursprung in der Tuberculose der Genitalorgane hätte. Ferner haben Autefaye und Dechert durch Operation bei einem 16 jährigen Mädchen tuberculöse Adnexe gefunden.

In der neuesten Literatur finden wir auch bei Frauen im vorgeschrittenen Alter Fälle von Genitaltuberculose. Hierbei ist noch der Fall von Joselin de Jong zu erwähnen, welcher bei der Untersuchung der Ausschabungsmassen einer 64 jährigen Frau krebsartige Elemente zusammen mit Tuberkelbacillen vorfand.

Wenn der Gynäkologe an dem Alter der Patientin keinen sicheren Anhaltspunkt für die Feststellung der Diagnose auf Genitaltuberculose hat, so stehen ihm betreffs der Vererbung nur wenige und in der Symptomatologie sehr wenige, meist gar keine Anhaltspunkte zur Verfügung.

Die allgemeinen und localen Symptome der Adnextuberculose unterscheiden sich nicht von denjenigen eines anderen bakteriologischen Ursprungs (Koblank), da in diesen das Krankheitsbild verschieden und frei sein kann auch von jenen Symptomen, denen man bei irgend einer anderen tuberculösen Affection begegnet (Ermattung, Abmagerung, Schweiss, Anorexie, Nachtfieber u. s. w.). Die Entwickelung des krankhaften Processes giebt uns keinerlei bestimmten Anhaltspunkt, was im Congress von Rom Faure zugab, mit dem Hinzufügen, dass die Adnextuberculose, im Besonderen, manchmal unmerklich entstehen und sich nach und nach entwickeln kann, ohne ernsthafte Schmerzen zu verursachen, mánchmal dagegen einen schnellen Verlauf nehmen und von acuten Adnexsymptomen begleitet sein kann. Wie Pozzi sagt, ist es eine der seltsamsten Erscheinungen der Genitaltuberculose, möglicher Weise 
316 Loiacono, Beitrag zur Tuberculose der weiblichen Adnexe.

lange Zeit latent und unerkannt zu blciben. Die isthmische, knollige Form, welche einige Autoren als von tuberculöser Natur ansehen möchten, trifft man auch bei Salpingitis von anderem bakteriellen Ursprung an. Die Verhärtung des Uterinhornes kann auch trügerisch sein.

Der bakteriologische Befund des Secrets lässt uns nicht immer den Tuberkelbacillus erkennen.

Die Hypoplasie der Genitalorgane soll nach der Meinung vieler Autoren (Amann, Truzzi, Pichevin, Merletti) ein gutes diagnostisches Symptom für die Tuberculoseaffectionen bilden. Besonders Merletti konnte bei 80 Individuen mit Genitalhypoplasie 24 mal Genitaltuberculose constatiren. Den genannten Autoren zufolge erzeugt die Uterinhypoplasie einen günstigen Boden für die Genitaltuberculose, entweder durch die Häufigkeit von Endometritis (nach Truzzi prätuberculär), oder durch die geringere Widerstandsfähigkeit, welche man in einem hypoplastischen Organ angeboren vorfindet, oder auch dadurch, dass sich in der Gebärmutterhöhle und im Tubenlumen jenes hemmende Secret leicht anhäuft, welches durch übertriebene und starre Anteflexion im Uterus und durch abnorme Krümmung in den Tuben gebildet wird.

In den letzten Jahren wurde von verschiedenen Gynäkologen die Tuberculinreaction als diagnostisches Mittel des Genitaltuberculoseprocesses erprobt, jedoch mit unbestimmten Resultaten.

Martin hatte keine guten Resultate, Birnbaum, welcher die Tuberculinreaction in 80 Fällen an der Göttinger Klinik versucht hat, fand das Gegentheil, bestätigt jedoch, dass, wo die Reaction gering ist, das Resultat zweifelhaft bleibt. Pankow hat die Tuberculinreaction ebenfalls in 22 Fällen erprobt, von denen 6 falsche Resultate ergeben haben, die anderen gute. Er schliesst damit, dass durch die Genitaltuberculose keine bestimmten Resultate erzielt wurden. Es ergiebt sich dann aus dem vorstehenden Berichte, dass die klinische Diagnose der Tuberculose in den weiblichen Genitalien meistens schwierig und manchmal unmöglich ist. Martin sagt mit Recht: "Bis jetzt kennen wir kein pathognomonisches Zeichen des Genitaltuberculoseprocesses".

Betreffs der Prognose der Genitaltuberculoseprocesse bei Frauen stimmen die Autoren darin überein, dass sie immer reservirt anzusehen ist.

Baisch berichtete im Jahre 1907 über 110 Fälle von Peritoneal- und Genitaltuberculose bei Frauen. Der zehnte Theil der 
Fälle wurde innerlich behandelt, die anderen chirurgisch. Von 110 Fällen starben 41 zwischen dem ersten und zweiten Jahre nach der Behandlung, die übrigen erlagen alle im Zeitraum von 4 Jahren. Wenn jedoch die Prognose unsicher ist, so hindert dies nicht daran, verschiedene Methoden zu erörtern und damit zu operiren, um, wenn nicht vollständig zu heilen, so doch das Leben der von Genitaltaberculose inficirten Patientinnen für eine mehr oder weniger lange Zeitdauer zu verlängern.

Was man vor allen Dingen zu rathen hat, ist die Prophylaxe der Genitaltuberculose, genau so wie die der Lungentubereulose (Stärkung der Reactionskraft des Organismus, Verminderung der Infectionsgefahr, Beeinflussung der prädisponirenden, schädigenden Momente (Gonorrhoe, Puerperium u. s. w.).

Die Verehelichung von Tuberculösen würde immer abzurathen sein (Veit und Hegar). Betreffs der Therapie neigt der grösste Theil der Autoren zur chirurgischen hin, besonders wenn die Genitaltuberculose nicht mit gänzlicher und sebwerer allgemeiner Tuberculose vereint ist. Ueber den besten Zeitpunkt zum operativen Eingriff, den zu beschreitenden Weg und die Ausdehnung der Operation bestehen die verschiedensten Meinungen.

Koblanck glaubt, dass bei einem beginnenden Tuberculoseprocess der chirurgische Eingriff nieht erforderlich ist, da eine spontane Heilung möglich sein kann, während um einen Herd zu zerstören, z. B. bei Tuben, man nicht vermeiden kann, dass Theile der inficirten Gewebe als Quellen der Infection zurückbleiben und beim Entfernen der Geschwulst von ihren Verwachsungen Eiter heraustreten und dadurch eine acute Peritonitis hervorgerufen werden kann.

Ebenso ist es in Tuberculosefällen, bei welchen eine Einkapselung des Herdes eintritt, nach Koblanck zwecklos zu operiren, weil in solchem Falle eine Verbreitung stattfinden könne.

Veit sagt, dass

1. Spontane Heilung von Tuberculose der Genitalien vorkommt.

2. Bei primärer und vielleicht auch bei isolirter Genitaltuberculose ist die Radicaloperation, wenigstens zur Zeit noch, die beste Heilungsmethode.

3. Bei nicht isolirter und secundärer Tuberculose der Genitalien kommt in erster Linie die allgemeine Therapie, vor allem Anstaltsbehandlung in Frage, doch kann man nicht verkennen, dass 
in einzelnen Fällen auch die Operation gute Dauererfolge erreicht. Sie kommt aber nur ausnahmsweise in Frage.

Martin ist ungefähr der gleichen Meinung, denn er giebt zu, dass bei weitgehender Erkrankung, besonders auch anderer Organe, die Therapie auf die Allgemeinbehandlung einzelner Symptome zu beschränken ist.: Ist der Process auf die Genitalien beschränkt, oder tritt die Genitalerkrankung zur Zeit in lebenbedrohender Weise in den Vordergrund, so bietet die Exstirpation des betreffenden Herdes eventuell der gesammten Genitalorgane Aussicht auf Erfolg und ist daher geboten.

Dagegen ist Pozzi der Meinung, dass man mit Totalhysterektomie in allen Fällen radical eingreifen müsse, ausgenommen in denjenigen, in welchen die begleitende Lungentuberculose in einem vorgerückten Stadium ist. In diesem Falle lässt sich nichts tuhn.

Faure hat dieselbe Ansicht wie Pozzi, dass man alle Tuberculoseherde zerstören müsse, und meint, die supravaginale Hysterektomie ausführen zu können, da die Tuberculose der Portio selten sei. In acuten Fällen sei er für einen Vaginaleingriff, weil auf diesem Wege die Drainage leichter sei. Pichevin dagegen meint, dass keinerlei Theile des Uterus, welche schon inficirt sein könnten oder die es später werden könnten, belassen werden sollen. Unter den von Pozzi bevorzugten Methoden befindet sich die von Terrier. Lea spricht sich therapeutisch für frühzeitige Entfernung und zwar beider Tuben aus, auch dann noch, wenn bereits das Peritoneum inficirt ist. Die Mitentfernung der Ovarien und des Uterus sei nicht generell anzuraten, sondern müsse von der jeweiligen Ausdehnung der Erkrankung abhängig gemacht werden.

Spinelli ist für eine conservative Therapie. Er will mit hypodermatischen Jodinjectionen die besten Resultate erzielt haben, sowohl bei operirten Patientinnen als auch bei Patientinnen, bei denen nur Jodbehandlung angewendet worden ist.

In den letzten Jahren sind bei tuberculösen Genitalaffectionen mit Koch'schen Tuberculinpräparaten Versuche angestellt worden. Birnbaum berichtet über bei derartigen Behandlungen erzielte Resultate. Von 7 Fällen von tuberculöser Peritonitis mit Ascites waren 3 geheilt worden, 2 blieben zweifelhaft, da der Autor sie lange Zeit nicht wieder beobachten konnte, 1 Fall wurde rückfällig, 1 anderer genas von der Peritonitis, bekam aber danach Knochentuberculose. Auf 7 Fälle ernster Peritonitis kamen fünf Heilungen, 1 Fall war auf dem Wege der Heilung, 1 Fall wurde 
nach einem Jahre rückfällig. Bei allen mit Tuberculin behandelten Patientinnen will Birnbaum Gewichtszunahme und Hebung des Allgemeinbefindens constatirt haben, wodurch sein Vertrauen zur generellen Behandlung gestärkt wurde.

Aus diesem Bericht über die wesentlichsten Abhandlungen der jüngsten Literatur ergiebt sich, dass unter den genannten Autoren nicht wenige Meinungsverschiedenheiten über viele Fragen bestehen.

Thatsächlich haben wir in Betreff der Aetiologie durch die verschiedentlichen experimentell erlangten Resultate und durch die verschiedenen Interpretationen, welche die Autoren ihren Fällen geben, noch keine bestimmten Angaben, auf Grund derer wir kurz und bündig versichern könnten, dass eine primäre weibliche Genitaltuberculose vorhanden sei.

Ebenso sind durch die Therapie die Meinungen über den richtigen Moment des Eingriffs, den geeignetsten bei der Operation zu befolgenden Weg und dessen Aúsdehnung verschieden.

Die Koch'schen Tuberculinpräparate, welche bei der Therapie der Genitaltuberculose der Frauen angewendet werden, setzen uns noch nicht in den Stand, bei einer solchen therapeutischen Methode vollkommen sicher zu gehen. Das letzte Wort hierüber kann erst dann gesprochen werden, wenn uns noch andere Resultate über diese wichtige Frage AufkJärung geben können.

Die Diagnose erscheint auch im gegenwärtigen Augenblick für den Gynäkologen sehwierig und ist oft nur durch das Mikroskop möglich. In letzter Zeit hat man Versuche damit angestellt, die Tubereulinreaction als Hülfsmittel bei der Diagnose anzuwenden, jedoch sind auch hier die Resultate gering und verschieden, auch wissen wir nicht, auf welche specielle Methode der Tuberculinreaction, die wie bekannt, unbeständig sind, man ein grösseres Vertrauen haben kann.

Wegen aller dieser auf dem Gebiet der Genitaltuberculose der Frauen auftauchenden Schwierigkeiten, darf ich wohl hoffen, dass die Darstellung der nachfolgenden 12 Fälle nicht vergeblich ist, da sie doch für die verschiedenen Ansichten mancher Forscher grösstentheils bestätigende Gesichtspunkte darbieten. Von den mir von Herrn Geheimrath Prof. Leopold in liebenswürdiger Weise zur Verfügung geste]lten 12 Fällen von Adnextuberculose gehören neun der Klinik, drei seiner Privatpraxis an.

Alle in Formalin gut conservirt gewesenen anatomischen Präparate der 12 operirten Patientinnen haben mir für die histologi- 
schen Untersuchungen die besten Dienste geleistet. Die Färbung ist mit Hämatoxylin-Eosin geschehen. Da der Nachweis von Kochschen Bacillen bei Präparaten älteren Datums sehr schwierig ist, habe ich hauptsächlich die typischen Tuberkeln und die Riesenzellen festzustellen gesucht, weil sie an sich genügend sind, die Diagnose der Tuberculose zu stützen.

Fall 1. 29. VI. 1898. N. N., 21 Jahre alt; verheirathet seit 1895. Beide Eltern und zwei Geschwister sind gesund. Mit 9, 14 und 18 Jahren Diphtheritis; als Kind Masern.

Erste Menstruation im 15. Jahre, schwach, 1 Tag dauernd. Ein $\mathrm{Jahr}$ aussetzend. Von da ab regelmässig; alle 4 Wochen, mittelstark, 5 Tage. Letzte Periode vor $21 / 2$ Wochen 8 Tage lang, unregelmässig; vorletzte 4 Wochen vorher. Früher Druck im Unterleib zur' Zeit der Periode; die letzten beiden Male obne jegliche Schmerzen. Keine Geburten und Fehlgeburten.

Jetzige Beschwerden: Seit ungefähr 7 Wochen stechende, nicht besonders starke Schmerzen in der rechten Seite des Unterleibs. Seit Frühjahr milchiger Ausfluss, der jetzt stärker geworden ist. Stuhl regelmässig; Harn 0 . B.

Schwächliche imittelgrosse Frau von gracilem Knochenbau und mässig entwickelter Musculatur. Gesichtsfarbe gesund. Leib etwas gespannt; Bauchdecken straff. Brüste mässig entwickelt, keine Secretion.

Portio zapfenförmig mässig geschwollen. Corpus uteri retroflectirt, nicht vergrössert. Beim Bewegen des. Uterus wird eine kleinapfelgrosse höckerige, mässig verwachsene Geschwulst nach links und binten fühlbar. Im rechten Parametrium lässt sich keine Geschwulst abtasten. Im Speculum zeigt sich die Vaginalportion hyperämisch. Aus dem Orificium externum quillt rein schleimiges klares Secret in geringer Menge.

Urin normal, ohne specifische Keime.

Klinische Diagnose: Retroflexio uteri, linksseitige Adnexerkrankung. Laparotomie am 15. VII. 98. Die ganze Tube ist in einen eiterhaltigen, Sack verwandelt mit zahlreichen Adhäsionen besetzt.

Ovarium kastaniengross mit Verwachsungen bedeckt. Schnittfäche zeigt zahlreiche bis bohnengrosse Cysten; Rinde stark verdickt. In Eiter Staphylococcus gyogenes albus. Im Tubenschnitt Riesenzellen und Tuberkelbacillen.

Diagnose: Salpingitis tuberculosa sinistra. Peritonitis tuberculosa.

Mikroskopischer Befund der linken Adnexe: Serosa der Tube bedeckt mit Auf lagerungen, die mit vielen neugebildeten Gefässen und Leukocyten, Tuberkeln mit Riesenzellen durchsetzt sind. Zwischen Serosa und Muscularis findet man eigenthümliche langgestreckte Spalträume, welche mit cubischem Epithel ausgekleidet sind. Die Muscularis ist locker und verdünnt, mit Leukocyten infiltrirt, ausserdem verdickte Gefässe.

Die Schleimhaut ist fast gänzlich zerstört, durchsetzt von vielen Leukocyten und käsigen Massen; in denselben befinden sich noch verdickte Schleimhautfalten, aber das Epithel ist ganz zu Grunde gegangen. Tuberkelknötchen und Riesenzellen befinden sich mebr in der Submucosa.

Ovarium von Auflagerungen bedeckt, kurze Strecken mit Resten von Keimepithel. In den Auflagerungen neugebildete Gefässe, Rundzellen- 
herde, Tuberkelknötchen mit Riesenzellen. In der Rindensubstanz viel Bindegewebe; einzelne der Primärfollikel geschrumpft. Die vorhandenen Follikel cystisch erweitert. Im Ovarialgewebe viele Gefässe mit Blut angefüllt.

Mikroskopische Diagnose: Salpingitis sinistra tuberculosa (käsige Form), Perioophoritis tuberculosa.

Am 18. VIII. 1908 nach Hause entlassen.

Fall 2. 9. X. 1898. N. N. 22 Jahre alt, verheirathet seit drei Jahren mit einem Manne, welcher wiederholt an Lungenblutungen gelitten hat und zur Zeit tuberculös ist. Opara.

Periode regelmässig, alle 4 Wochen 3 Tage lang.

Die Patientin hat fortwährend Schmerzen im Unterleib. Ans der Beckenhöhle wölbt sich links eine unregelmässige Geschwulst hervor, welche als Pyosalpinx angesprochen wird. Laparotomie am II. XI. 1898. Die linke Tube zeigt am uterinen Ende fast normale Beschaffenheit, verläuft dann auf $5 \mathrm{~cm}$ bleistiftdick und biegt dann spitzwinkelig in einen hühnereigrossen Eitersack um. In dem spitzen Winkel sitzt das wenig vergrösserte Ovarium, das ringsum kleine Cysten durchschimmern lässt. An der Oberfläche einige derbe Adhäsionen.

Die rechten Adnexe bilden ein gänseeigrosses Packet, das ringsum mit zahlreichen, zum Theil derben Adbäsionen besetzt ist. Die Tube verläuft ganz ähnlich wie die linke, vom Fimbrienende jst nichts zu sehen. Das Ovarium ist bis auf das Dreifache des Normalen vergrössert. Die Oberfläche des Ganzen äusserst uneben, mit vielen grösseren und kleineren bis stecknadelkopfgrossen Knötchen besetzt

Diagnose: Pyosalpinx daplex. Perisalpingitis et Perioophoritis chronica duplex tuberculosa.

Mikroskopiscber Befund: Im Uterinende der linken Tube ist die Mucosa fast normal, die Submucosa wenig von Leukocyten infiltrirt. Die musculäre Schicht stark durchsetzt von Rundzellen mit Tuberkelknötchen und Riesenzellen. Die Serosa verdickt mit wenig Verwachsungen.

In der Mitte der Tube ist die Schleimhaut mässig infiltrirt von Leukocyten, aber das Epithel ist gut erhalten. In der submucösen und in der musculären Schicht starke Infiltration, Detritusmassen, Tuberkelknötchen, viele Riesenzellen. Zahlreiche Gefässe sind in der Serosa und Muscularis vorhanden. Serosa verdickt, infiltrirt.

Am Abdominalende ist die Musculatur sehr dünn, infiltrirt von Rundzellen, nach der musculären Schicht käsige, structurlose Detritusmassen vermiscbt mit Blut, die das ganze Tubenlumen durchsetzen.

Linkes Ovarium: Mässige Verwachsungen auf der Oberfläche; das Keimepithel ist noch auf einer kurzen Streeke vorhanden. In der Rindensubstanz zahlreiche primäre, Graaff'sche und cystische Follikel. Im Ovarialstroma zahlreiche Gefässe und Corpora albicantia.

Rechte Tube: Serosa verdickt mit Auflagerungen, sehr infiltrirt. Musculatur verdickt und von Leukocytenherden und Tuberkelknötehen durchsetzt. Die Mucosa fast ganz zerstört bis auf einzelne Schleimhautreste, besteht aus Leukocyten, käsigen Massen mit vielen Riesenzellen. Rechtes Ovarium: Anf der Oberfäche gefässreiche Verwachsungen. Im Bindegewebe sind einzelne degenerirte Primärfollikel vorhanden und mässige Rundzelleninfiltration.

Im Ovarialstroma starke Rundzelleninfiltration, zahlreiche Gefässe, viele Tuberkelknötchen mit Riesenzellen. 
Mikroskopische Diagnose: Salpingitis sinistra et dextra tuberculosa (käsige Form). Perioophoritis sinistra et Oophoritis dextra tuberculosa (mildere Form).

Fall 3. 15. VII. 1898. N. N., 25 Jahre alt; verheirathet seit: 1 Jahre. Keine Geburt. Letzte Periode vor fast 3 Wochen.

Erkrankt am 25. Juli 1898 unter heftigen Schmerzen und hohem Fieber an Metritis uud Perimetritis. Das mässige Exsudat füllte am nächsten Tage die Höhle des kleinen Beckens aus, und unter schweren allgemeinen Erscheinungen verlief die Krankheit, bis anfangs September, wo erst eine Resorption nachweisbar war. Während der Krankbeit war die Periode vollständig weggeblieben und trat nach derselben, regelmässig 4 Tage dauernd, auf. Zur Zeit handelt es sich um eine straff fixirte Retroflexio uteri. Laparotomie am 4. V. 1899. Die rechte Tube kleinfingerdick mit derben Adhäsionen bedeckt; das Fimbrienende verlöthet. Wand stark verdickt, obne Inhalt. Dicht an ihr sitzt das gut hühnereigrosse Ovarium, ebenfalls mit derben Verwachsungen besetzt. Auf dem Durchschnitt sieht man das ganze Gewebe durchsetzt von Eiter und Blut. Die linke Tube jst fast ebenso dick wie die rechte, sie ist mit dem Ovarium zu eineı gut wallnussgrossen Packet verlöthet. Das Gewebe ist blasig durchtränkt, cystisch degenerirt. Das Ganze ebenfalls mit derben Adhäsionen besetzt.

Diagnose: Chronische Salpingitis et oophoritis duplex tuberculosa.

Mikroskopischer Befund: Linke Tube von Verwachsungen bedeckt. Die Musculatur verdickt und mit Rundzellen durchsetzt. Tubenlumen vollständig ausgefüllt von käsiger Masse, in welcher noch Epithelreste zu seben sind.

Linkes Ovarium: Auf der Oberfläche einzelne Verwachsungen, aber einze!ne Strecken mit Keimepithel bedeckt. Im Ovarialstroma einzelne cystisch erweiterte Follikel und viele Gefässe.

Rechte Tube: Auf der Oberfläche zahlreiche Adbäsionen mit vielen Gefässen und Leukocyten durchsetzt. Musculatur verdickt und rolk zahlreicher Rundzellenherde; geringe Tuberkelknötchen und Riesenzellen sind vorhanden.

Rechtes Ovarium: Im Gewebe zahlreiche Gefässe, Luteinzellen und cystisch erweiterte Follikel. Stroma besteht aus käsiger Hlasse und enthält einzelne Riesenzellen.

Diagnose: Salpingitis tuberculosa sinistra (Form - caseosa), Salpingitis tuberculosa dextra, Perioophoritis tuberculosa sinistra, Oophoritis dextra.

Die Patientin hat sich sehr gat erbolt und bei der letzten Untersuchung vor 2 Jahren sehr wohl befunden.

Fall 4. 13. IV. 1900. N. N., 23 Jahre alt, verheirathet seit 6 Monaten.

Ueberstandene Krankheiten: Masern, Scharlach, Bleichsucht im 16. Jahre. Im 15. Jahre erste Periode, regelmässig, stark. Letzte Periode vor 14 Tagen. Eine Spontangeburt, Kind lebt, gesund. Vor acht Wochen merkte Patientin, dass ihr Leib grösser wurde, keine Blutangen. Dabei auch Schmerzen im Unterleib und allgemeine Schwäche. Appetit gut. Stuhl regelmässig. Harn normal.

Habitus tuberculosus. Herz verbreitert. Geräusch an dem I. Aortenton. Deutliche Tachycardie. Lungenschall weniger sonor an der 
linken Spitze als an der rechten. Bauchdecken sehr dünn und straff. Brüste gut entwickelt, kein Secret.

Vulva und Vagina o. B., Portio aufgelockert. Uterus klein, steht rechts und vorn. Die Dämpfungsgrenzen werden nur wenig verschoben bei der Seitenlage. Keine Fluctuation im Becken za fühleu.

Im Vaginal- und Urethralsecret keine specifischen Keime.

Diagnose: Peritonitis ehronica tuberculosa.

Laparotomie: 19. IX. 1900 . Tube $5 \mathrm{~cm}$ lang, am Uterinende normal, nach dem abdominalen Ende wie ein kleiner Finger zu einem Packet zusammengehalten, von hochrother Farbe mit sehr zahlreichen weissen Knötchen übersät. Fimbrienkranz erhalten, sebr stark entwickelt und ebenfalls mit Knötchen übersät. Fimbria ovarica gat erhalten, führt auf das Ovarium. Auf den Durchschnitt erscheint die Tubenwand stark verdickt bis $3 \mathrm{~mm}$. Das Lumen ist stricknadelstark an der stärksten Auftreibungsstelle und von einer weisslichen festen Masse angefüllt. Das linke Ovarium kleinpflaumengross, mit weissen Knötchen übersät, leicht höckerig, viele zarte Verwachsungen. Auf dem Durchschnitt erscheint die Rinde etwas verdickt, man findet einige linsenbis erbsengrosse Cysten (Follikel).

Diagnose: Perioophoritis et perisalpingitis tuberculosa.

Mikroskopischer Befund: Linke Adnexe. Salpinx: Auf der Oberfläche viele Verwachsungen. Musculatur ist verdickt und blutig serös durchtränkt; man findet starke Infiltration und Tuberkelknötchen mit Riesenzellen.

Das ganze Lumen ist vollständig ausgefüllt von Schleimhantzotten, welche blutig-serös durchtränkt sind. Im Zottenstroma wenige Leukocyten.

Orarium: In der Rindensubstanz sind Primärfollikel and einzelne cystisch erweiterte Follikel vorbauden. Das Ovarialstroma ist ganz normal.

Diagnose: Salpingitis tuberculosa sinistra (miliare Form), normales Ovarium.

Die Patientin ist am 14. X. 1900 obne subjective Beschwerden entlassen.

Fall 5. Aufgenommen am 21. V. 1902. N. N., 28 Jahre alt, verheirathet seit 5 Jahren, keine Geburt. Leidet seit 4 bis 5 Jahren an starker Blutung und Schmerzen im kleinen Becken. Nach dem Sondenbefund handelte es sich um eine Endometritis polyposa. Es wurde nach Erweiterung mit Laminaria am 26. V. 1902 eine Ausschabung vorgenommen, an welche sich eine exsudative Parametritis anschloss. Das Exsudat wurde am 7. Juni in der Klinik eröffnet, noch im October 1902 war ein grosser Exsudatrest, namentlich links, zu fühlen, welcher im December 1902 dem Durchbruch nach dem Rectum nahe zu sein schien. Da aber dieses Exsudat nicht durchbrach, so wurde am 13. T. 1903 die Laparotomie vorgenommen und die beiderseitigen, sehr schwer erkrankten und mit Eiter erfüliten Adnexe entfernt.

Pathologischer Befund: Linke Tube stark verdickt und geschlängelt. Auf der Oberfläche dicke fibröse Auflagerungen. Fimbrienenden mit dem Ovarium vollständig verwachsen. Serosa und Schleimhaut stark geröthet. Tubenlumen leer, federkielweit. Ovarium hühnereigross mit höckeriger Oberfläche und fibrösen Auflagernngen. Der mediale, mit der Tube verwachsene Pol des Ovariums ist bei der Ope- 
ration eingerissen infolge der dünnen, durch den Eiter der Abscesshöble arrodirten Wandung, die sehr brüchig ist. Innenfläche der Höble rauh, mit Fibrinbeschlägen ganz ausgekleidet. Dicke der Wandung ungefähr $5 \mathrm{~mm}$.

Rechtes Ovarium über hübnereigross. Obcrfläche uneben, mit Fibrin bedeckt. Tube ist mit dem Ovarium vollständig verlöthet. Fimbrienenden geschlossen. Tubenlumen stark kleinfingerweit, mit Eitermasse ausgefüllt. Schleimhaut wenig gefaltet. Schnittläche des Ovariums von grau-gelber Farbe; nur in der Mitte zeigt sich leichte reactive Röthung des noch vorhandenen Stromagewebes.

Diagnose: Pyovarium duplex. Pyosalpinx dextra. Linksseitige chronische interstitielle Salpingitis.

Mikroskopischer Befund: Linke Tube: Musculatur ist wenig von Leukocyten durchsetzt, viele erweiterte Lymphspalten. Die Mucosa ist gänzlich zerstört, besteht aus Käsemasse mit vielen Riesenzellen und Tuberkelknötchen.

Linkes Ovarium: Auf der Oberfläche Verwachsungen. Die Rindensubstanz ist locker, mässig infiltrirt. Das Ovarialstruma besteht aus Detritusmasse und polynucleären Lenkocytenberden, durchsetzt von Tuberkelknötchen mit Riesenzellen.

Rechte Tube: Auf der Oberfäche viele Verwachsungen mit zahlreichen Gefässen und mässigen Rundzellen.

Die musculäre Schicht ist sehr dick, ziemlich viele Gefässe, mässige Leukocyten.

Die Schleimhaut ist etwas gesehwollen. Die Zotten untereinander verwachsen und bilden Cysten, welche mit seröser Flüssigkeit gefüllt sind. Einzelne Zotten tragen noch Epithel. Geringe Leukocyteninfiltration.

Rechtes Ovarium: Die Innenfläche besteht aus einer Abscesshöhle, in welcher sich fluctuirende und fest anhaftende Käsemassen mit Tuberkelkuötchen und Riesenzellen befinden. Die Abscesswand besteht aus Granulationsgewebe mit vielen kleinen neugebildeten Gefässen und zahlreichen polynucleären Leukocyten. Zwischen Abscesswand und Rindensubstanz liegt eine stark infiltrirte Zone. Die Rindensubstanz selbst ist locker, mässig infiltrirt. Auf der Oberfläche wenig Verwachsungen.

Mikroskopische Diagnose: Salpingitis sinistra tuberculosa (käsige Form). Salpingitis dextra tuberculosa (miliare Form). Oophoritis sinistra tuberculosa (käsige Form). Oophoritis dextra tuberculosa (abscedirende Form).

Obwohl sich die Kranke von dem Eingriff bald erholt hatte, erkrankte sie zu Hause später, nach ihrer Angabe an Lungenentzündung und bot bei der letzten Untersuchung am 24. Juli 1903 die Erscheinungen der Phthisis pulmonum dar.

Fall 6. No. 99. 27. III. 1903. N. N., 33 Jahre alt, verheirathet seit $10^{1 / 2}$ Jahren.

Vater an Altersschwäche gestorben, Mutter und Geschwister leben und sind gesund.

Als Kind Masern, im Alter von 27 Jahren Diphtheritis.

Erste Menstruation im 14. Jahre, immer regelmässig, nie schmerzhaft bis zum 24. December. Seitdem vor Beginn der Menstruation heftige, nach unten zusammenziehende Schmerzen. Nullipara.

Patientin will seit 20. XII. 1902 stechende Kopfschmerzen und Schmerzen in der linken Seite haben, die zuerst nur während der Periode 
auftraten, seit 3 Wochen aber unaufhörlich sind. Irgend ein Trauma etc. weiss sie nicht anzugeben. Appetit schlecht; Stuhl regelmässig; Harn: Stechende Schmerzen beim Uriniren.

Kleine, gracil gebaute, schlecht genährte Frau von kränklichem Aussehen. Lymphdrüsen ohne Befund. Herztöne rein, leise. Lunge gesund. Zunge etwas weisslich belegt. Darm hat normalen Schall. Leib $63 \mathrm{~cm}$ Umfang in Nabelhöhe; $14 \mathrm{~cm}$ von der Symphyse bis Nabel; $15 \mathrm{~cm}$ vom Nabel bis Proc. xiphoideus. - Vaginalschleimhaut rauh, Portio etwas geschwollen. Aus der Cervix quilit grüngelbes Secret. Uterus nicht vergrössert, anteflectirt, beweglich. Links von ihm sitzt ein für sich abgrenzbarer, gut mannesfaustgrosser, ziemlich fester Tumor, der den linken Adnexen entsprechen dürfte. Das rechte Ovarium ist als kastaniengrosser Tumor fühlbar.

In dem mikroskopischen Befund des Secretes finden sich Kokken und Diplokokken. Diagnose: Endometritis, linksseitiger Adnextumor, rechtsseitige Oophoritis.

Laparotomie am 31. III. 1903: Rechte Tube, $5 \mathrm{~cm}$ lang, geschlängelt und verdickt. Oberfläche glatt, ohne Adhäsionen, stark geröthet. Fimbrienende an der grössten Peripherie mit dem Ovarium verwachsen. Lumen verschlossen. Musculatur $1 / 2 \mathrm{~cm}$ verdickt. Schleimhaut geschwollen und geröthet.

Rechtes Ovarium hühnereigross, grünlich-roth, stellenweise blänlichroth. Oberfläche grösstentbeils glatt, nur wenig fibrinöse Auflagerungen. Consistenz weich. Der ganze Eierstock besteht aus einer Abscesshöhle, deren Wandung $1 / 2-3 / 4 \mathrm{~cm}$ dick ist und nur noch ganz wenig normales, blass röthliches Ovarialstroma enthält. Die Innenfläche der Abscesswandung ist stark zerklüftet von grünlich-gelber Farbe, und mit eitrig fibrinösen, nicht abstreifbaren Auflagerungen bedeckt. An der vorderen oberen Fläche ist eine markstückgrosse Perforationsöffnung, die während der Operation einriss. Der Eiter ist gelb, dünuflüssig.

Linke Tube $6,5 \mathrm{~cm}$ lang, verdickt geschlängelt. Die Verdickung am abdominalen Ende der Tube beträgt $1,5 \mathrm{~cm}$. Aus dem verschlossenen Fimbrienende ragt ein kirschgrosser, glattwandiger, derber Knoten hervor. Oberfläche der Tube stark geröthet, mit bindegewebigen Adhäsionen bedeckt. Auf der Schnittfäche zeigt sich die Wandung nur $1,5 \mathrm{~mm}$ dick, das Lumen ist mit gelblich-grünem Eiter ausgefüllt. Der oben erwäbnte Knoten stellt eine von einer Bindegewebskapsel umgebene Eiteransammlung dar.

Linkes Ovarium pflaumengross; Oberfläche zeigt viele narbige Einziehungen und wenig bindegewebige Adhäsionen. Farbe grünlich-blau, Consistenz fest. Albuginea nicht verdickt. Das blassrothe, derbe Gewebe der Schnittfläche ist durchsetzt von mehreren linsen. bis erbsengrossen, glattwandigen, mit klarer seröser Flüssigkeit ausgefüllten Cysten. Gefässreichthum des Ovarialstroma etwas vermehrt.

Diagnose: Salpingitis et Pyovarium dextrum, Pyosalpinx sinistra. Chronische Oophoritis mit Cystenbildung links.

Mikroskopischer Befund: Linke Tube verdickt, in der Muscularis starke Infiltration und zahlreiche mit Blut gefüllte Gefässe.

Die Mucosa ist ganz zerstört und besteht aus vielen Tuberkelknötchen mit Riesenzellen. Das Lumen ist erweitert.

Linkes Ovarium keine Verwachsungen. In der Rindensubstanz cystisch erweiterte Follikel.

Im Ovarialstroma viele Corpora albicantia, zahlreiche Gefässe mit 
verdickten Wandungen, dickliches und ödematöses Bindegewebe, welches von zahlreichen embryonalen Zellen durchsetzt ist.

Rechte Tube mit vielen Verwachsungen und zahlreichen Gefässen. Die Musculatur ist mässig verdickt.

Die Falten der Schleimhaut sind miteinander verwachsen, sie sind von zahlreichen Rundzellen und einzelnen Tuberkelknötchen mit Riesenzellen durchsetzt. Rechtes Ovarium zeigt dasselbe Bild, wie das rechte Ovarium des Falles 5.

Mikroskopische Diagnose: Salpingitis sinistra 'tuberculosa (käsige Form), Salpingitis dextra tuberculosa (miliare Form), Oophoritis interstitialis sinistra, Oophoritis dextra (abscedirende Form).

Geheilt entlassen am 30. V. 1903.

Fall 7. No. 292. N. N., 27 Jahre alt, verheirathet seit 3 Jahren. Eltern und Geschwister leben und sind gesund.

Als Kind gesund, ab und zu Magenbeschwerden.

Erste Ienstruation im 15. Jahre, regelmässig. Letzte Periode am 28. VI: 8 tägig stark; die letzten 3 Perioden stärker als früher. Keine Geburten und Fehlgeburten. War wegen starker Periode in ärztlicher Behandlung. Klagt über Stechen in der linken Seite, Ziehen im Kreuz. Wenig Fluor. Ist vom Arzt massirt worden, hat aber mit Fieber reagirt. Arzt setzte daher die Massage aus. Appetit mässig, Stuhl letzte Zeit regelmässig; öfters Harndrang, Gesichtsfarbe und übrige Haut biass.

Lymphdrüsen ohue Befund. Herzgrenzen nicht erweitert; an der Spitze ist ein leises Geräusch mit dem I: Ton zu hören; dasselbe verschwindet aber beim Aufsitzen der Patientin. Ueber der linken Clavicula ist der percutorische Schall gedämpft; einzelne Rhonchi.

Verdaunngscanal ohne Befund. Leibesumfang in Nabelböhe $67 \mathrm{~cm}$; Symphyse bis Nabel $18 \mathrm{~cm}$; Nabel bis Proc. xiphoideus $15 \mathrm{~cm}$. Brüste sehr schlaff. Vulva geschlossen; Vagina sehr eng. Vaginalportion derb, Uterus nicht vergrössert, anteponirt. Vom Uterus nach unten eine den Douglas ausfüllende, höckerige, derbe, wenig schmerzhafte Geschwulst, welche nach links und rechts fast bis an die Beckenwand reicht. Adnexe sind nicht zu fühlen. Per rectum fühlt man, dass der Tumor die vordere Rectalwand nach dem Kreuzbein zu drückt, so dass das Lumen an dieser Stelle sehr flach ist.

Diagnose: Retropositio et retroflexio uteri; linksseitige Adnexerkrankung.

Laparotomie am 6. VIII. 1903. Linke Tube $8 \mathrm{~cm} \mathrm{lang,} \mathrm{am} \mathrm{Uterin-}$ ende federkieldick, am Fimbrienende bis dammendick, stark geschlängelt und geröthet, von reichlichen Adhäsionen bedeckt. Fimbrienende offen. Das Lumen kleinfingerdick, vollkommen ausgefüllt mit Eiter; Wand stark verdickt. Linkes Ovarium mandelgross, glatte Oberfläche, zahlreiche Adhäsionen; an der Oberfläche zahlreiche Cysten durchschimmernd; auf der Schnittfäche gleichfalls zahlreiche Cysten; Gewebe geröthet.

Rechte Tube $7 \mathrm{~cm}$ lang, am uterinen Ende federkieldick, nach dem Fimbrienende stark anschwellend, zeigt im Uebrigen dieselben Verhältnisse wie links. Fimbrienkranz erhalten. Tubenlumen mit Eiter angefüllt.

Rechtes Ovarium hühnereigross, glatte Oberfläche, reichliche Ver- 
wachsungen, stark geröthet. Auf dem Durchschnit eine wallnussgrosse, mit Eitermassen ausgefüllte Höhle; Wand derselben 5-6 mm dick.

Diagnose: Pyosalpinx duplex, Pyovarium dextrum. Degeneratio cystica ovarii sinistri.

Nlikroskopischer Befund: Linke Tube: Auf der Oberfäche viele Verwachsungen mit zahlreichen neugebildeten Gefässen. Die musculäre Schicht ist dünn, durchsetzt von zahlreichen Leukocyten. Das Tubenlumen ist stark erweitert; die Schleimhautzotten platt gedrückt. Das Lumen besteht aus błatiger seröser Flüssigkeit mit zahlreichen Leukocyten. Das Zottenepithel ist ganz zu Grunde gegangen. In den Schleimhautresten siad Riesenzellen und Tuberkelknötchen vorhanden. Linkes Ovarium: Auf der Oberfläche zarte Verwachsungen. Im Rindengewebe Primordial- und cystische entartete Follikel. Im Stromagewebe sind zablreiche Gefässe und Corpora albicantia vorhanden.

Recbte Tube: Auf der Oberfläche zablreiche Verwachsungen mit vielen Gefässen und Rundzellen.

Die musculäre Schicht ist dünn, zahlreiche Leukocytenherde. Das Tubenlumen ist stark erweitert, von blutig seröser Flüssigleit angefüllt. Die Schleimhaut ist platt gedrüclst und von vielen Riesenzellen durchsetzt. Das Zottenepithel fehit. Rechtes Ovarium: Mässige Verwachsungen auf der Oberfläche. Das Ovarialgewebe ist blutigserös durchtränkt. Im Rindengewebe befinden sich einzelne Primärfollikel und Graaf'sche Follikel, welche zum Theil in Schrumpfung übergehen. Luteinzellen sind auch vorhanden. Das Ovarialstroma ist von Käsemasse, Tuberkelknötchen mit Riesenzellen und Eiter durchsetzt.

Diagnose: Salpingitis sinistra et dextra tuberculosa (käsige Form). Perioophoritis sinistra et oophoritis dextra tuberculosa (käsige Form).

Am 15. VIII. 03 Exitus letalis.

A utopsie: Bronchialdrüsen verkäst. Lungenembolie.

Fall 8. No. 53. 31. 1. 1907. N. N., 37 Jahre alt, verheirathet seit 1895. Vater starb 59 Jahre alt, Mutter 44 Jahre alt an Herzerweiterung; 4 Geschwister leben. 1 Schwester starb mit 32 Jahren an Lungentuberculose.

Als Kind Diphtherie und Scharlach. Im Jabre 1901 lag die Kranke 4 Monate lang wegen Mutterbandentzündung im Krankenhars. Am 6. X. 1905 wurden ihr in der hiesigen Klinik die linken Adnexe entfernt. (Nach dieser Operation blieb in der Bauchwunde eine Fistel vorhanden.)

Wit 18 Jahren erste Menstruation, dann blieb die Periode 1 Jahr lang aus, dann kam sie wieder regelmässig, alle 4 Wochen. Letzte Regel vom 21.-26. I. 1907. Nullipara. Jetzige Beschwerden: Schmerzen im Unterleib, Fistel in der alten Bauchwunde, weshalb die Frau sich operiren lassen will. Die Fistel verursachte manchmal brennende Schmerzen.

Appetit gut; Stuhl angebalten; Harn ohne Befund.

Mässig kräftige Frau im mittleren Ennäbrungszustande. Gesichtsfarbe blass. An beiden Beinen Varicositäten. Lymphdrüsen nicht geschwollen. An Herz und Lungen keine krankhafien Veränderungen.

In der Linea alba von Nabel bis. Mons pubis senkrecht verlaufend alte weisse Operationsnarbe, in der Mitte von strahliger Beschaffenheit. Ueber dem Mons pubis besteht eine offene secernirende Stelle. Brüste; keine Secretion. Vulva und Vagina sind weit. Portio virginell; äusserer Muttermund punktförmig. Uterus klein, anteflectirt, beweglich. 
Linkes Parametrium etwas verdickt. Rechtes Ovarium vergrössert, deutlich fühlbar, ebenso Tube als dicker Strang fühlbar.

Urin normal. Im Vaginal- und Urethralsecret befinden sich viele Kokken und Stäbchen, Epithelien und wenig Leukocyten.

Diagnose: Salpingitis und Oophoritis tuberculosa dextra, Bauchdeckenfistel.

Laparotomie am 15. VI. 1907. Linke Adnexe im Jahre 1905 entfernt. Linke Tube $3 \mathrm{~cm}$ lang, stark verdickt und geröthet, mit vielen breiten Verwachsungen. Fimbrien schwerer verändert. Lumen nur als undurchgängiges Grübchen erhalten. Starke Verdickung und Röthung der Wand, aus dem Lumen quillt bräunliches Blut hervor.

Linkes Ovarium kastaniengross; Hinterfläche missfarbig, grünlich, uneben, mit vielen Verwachsungen; Vorderfläche gelblich, auch mit vielen Verwachsungen bedeckt. Rinde nicht verdickt. Ovarialgewebe stark injicirt. Keine Cysten; ein altes Corpus luteum ist vorhanden.

Rechte Adnexe entfernt bei zweiter Operation.

Die Tube ist $3 \frac{1}{2} \mathrm{~cm}$ lang, verdickt, roth, mit zarten Verwachsungen, Fimbrienkranz ist verklebt, Lumen geschlossen. Das Ovarium ist $44_{2}^{1 / 2}: 3^{1 / 2}: 2 \mathrm{~cm}$ dick. Oberfä̈che uneben, mit vielen Verwachsungen von theils blauer theils grïner Farbe. Am uterinen Pol eine haselnussgrosse Blutcyste; durch die Oberfläche schimmern viele Cysten.

Diaguose: Perisalpingitis und Perioopboritis sinistra, Salpingitis dextra, Degeneratio cystica ovarii dextri.

Mikroskopischer Befund: Linke Tube: Auf der Oberfläche zahlreiche Verwachsungen. Die musculäre Schicht ist sehr dünn und locker, von vielen kundzellen und Gefässen von verdickter Wandung durchsetzt. Tubenlumen ist erweitert, von Käsemasse ausgefüllt. Die Schleimhautfalten sind theilweise plattgedrückt und von Riesenzellen durchsetzt, theilweise fehlt das Epithel.

Linkes Ovarium: Auf der Oberfläche Verwachsungen. In der Albuginea befinden sich isolirte Tuberkelknötchen mit Riesenzellen. Die Rindensubstanz und das Ovarialstroma zeigen normales Gewebe mit hydropischen Follikeln und Corpora albicantia.

Rechte Tube: Mässige Verwachsungen auf der Oberfläche. Die musculäre Schicht verdickt mit zahlreichen Gefässen, ist wenig infiltrirt. Im Tubenlumen sind die Schleimhautzotten miteinander verwachsen und bilden Cysten, in welchen eine käsige Masse liegt. Die Schleimhaut ist von vielen Tuberkelknötchen und Riesenzellen durchsetzt.

Rechtes Ovarium: In der Rindensubstanz normale Primärfollikel und Follikelcysten. Im Ovarialstroma zahlreiche Gefässe, ein Corpus luteum und Corpora albicantia.

Diagnose: Salpingitis sinistra et dextra tuberculosa (käsige Form), Perioophoritis sinistra tuberculosa. Rechtes Ovarium normal.

Die Patientin hatte in den letzten Tagen guten Appetit und guten Schlaf. Der Koth entleert sich täglich aus der zweimarkstückgrossen granulirenden Fistel und auf natürlichem Wege. Es besteht Harndrang. Die Frau füblt sich sehr matt und klagt über Leibschneiden (sonst subjectives Befinden leidlich gut).

Wird mit Verband entlassen.

Fall 9. No. 325. 2. VII. 1908. N. N., 46 Jahre alt, verheirathet seit 1886. Vater an Herzschlag, Mutter an Gebärmutterkrebs, ein Bruder an Darmverschlingung gestorben, eine Schwester lebt. 
Die Patientin hatte Scharlach, Bleichsucht, Gebärmutterentzündung bis 1885 und im Jahre 1901 Hauterkrankung 2 Monate lang, im Jahre 1906 Blasen an den Füssen, Zellgewebsentzündung (?) am linken Arm. Erste Regel mit 12 Jahren, 4 wöchentlich, 7 Tage lang und stark, meist flüssiges Blut, zeitweise auch geronnenes. In den ersten drei Tagen stets kolikartige und stechende Schmerzen in der Mittellinie des Unterleibes.

Letzte Regel am 9. VI., 6 Tage lang, sehr stark.

Eine Fehlgehurt im 4. Monat (1886). Die Menstruationsschmerzen treten meist in der Gegend der Gebärmutter auf, manchmal ziehen sie auch durch den ganzen Leib. Seit der Menstruation im Mai 1907 bestanden täglich 2-5 Stunden lang abwechselnd diese Schmerzen, die aber seit der letzten Regel im Juni vollständig verschwunden sind.

Dr. P. schickte sie wegen Entartung des Mutterbalses hierher (fand in Probeexcision Carcinom). Patientin sehr anämisch. Varicen der Beine. Herzgrenzen normal, Töne rein. Lungenschall bell. Athmung vesiculär, Zunge trocken, weisslich verfärbt. Bauchdecken fettreich; Palpation nirgends schmerzhaft. Absturz beiderseits vorhanden, Leib ziemlich voll; nirgends Dämpfung. Brüste nicht vergrössert, keine Secretion. Vulva obne Befund. Vagina eng. Portio ziemlich zerklüftet in der Mittellinie; Uterus in gleicher Stellung, anteflectirt, leicht beweglich; rechtes Parametrium frei, das linke nicht ganz frei. Die Rauhigkeit der Portio geht etwas auf das linke Scheidegewölbe über.

Speculumuntersuchung: Portio sehr verkürzt, in ein festes, leicht blutendes Gewebe umgewandelt. Muttermund sehr eng, der einer Nullipara. Sonde lässt sich einführen.

Urin normal.

Diagnose: Carcinoma portionis.

(Die fetten Bauchdecken ungünstig wegen Gefahr der Infection des Fettgewebes mit Carcinom bei abdomineller Operation. Abwarten der in der nächsten Woche in Aussicht stehenden Venstruation, dabei Bettrube, Eisbeutel, Stypticintabletten). Patientin erkrankte hierauf an einer linksseitigen Pleuritis, nach deren Heilung sie fulgurirt wurde, weil der Uterus nicht ganz beweglich war. Nach 1 Monat war die Beweglichkeit des Uterus gebessert und die Patientin wurde operirt.

$\mathrm{Am}$ 3. XI. 1908 Laparotomie. Die beiden Adnexe wurden nur entfernt; der Uterus ]ässt sich nicht exstirpiren, weil die vordere Uteruswand mit der Blase stark verwachsen ist.

Pathologischer Befund: Rechte Tube $8 \mathrm{~cm}$ lang, geschlängelt, stark geröthet, bis kleinfingerdick mit zahlreichen Verwachsungen. Reste vom Fimbrienkranz erbalten, Lumen mit dem Ovarium verklebt. Dicht am Fimbrienkranz nach dem Uterus zu eine taubeneigrosse durchscheinende Cyste in der Wand der Tube. Lumen ist zu erkennen, mit derbem, glasigem, weissgelbem Gewebe ausgefüllt, das von Hohlräumen durchsetzt ist. Die Wand ist verdiekt, dunkel grauröthlich, mit kleinen Gefässen. Ligamentum latum verdickt, geröthet.

Recbtes Ovarium $3: 3: 2 \mathrm{~cm}$, blassgelb, mit Verwachsungen auf der Oberfläche, die narbig eingezogen ist. Mehrere Cysten liegen unter der Oberfläche, von denen eine mit blutigem Inhalte und dünner Wand besonders hervortritt.

Linke Adnexe: Apfelgrosses Packet von unregelmässiger Form. Die Oberfläche ist theilweise glatt, da, wo sie mit Serosa überzogen ist. An der Hinterseite ist sie stark zerklüftet. Das zu Tage tretende Gewebe 
sieht theils markig, theils eitrig infiltrirt aus. Aus einigen Buchten lässt sich eitrige Masse herausdrücken. Die Serosa ist geröthet und mit Verwachsungen bedeckt. An der Oberkante des Packets läuft die verdickte Tube dicht aufsitzend, fingerdick. Sie hebt sich von dem Packet wenig ab, da die Serosa beide straff überzieht, so dass eine Furche dazwischen läuft. Tube ist ausserdem geschlängelt. Das Ganze ist derb, besonders die Tube. Fimbrienkranz und Lumen nicht zu sehen. Auf dem Durchschnitt läuft aussen herum die Tubenwand zu fast $2 / 3$ des Umfanges des Packets. In der Mitte das theils markig-derbe Consistenz zeigende, theils eitrig erweichte Ovarium. In der Mitte eine längliche Höhle, deren Wände gräulich-gelblich bröcklig sind. Tube und Ovarium sind eng miteinander verschmolzen. Das Tubenlumen ist mit einer derben, glasigen Masse ausgefüllt, die mit der Wand eng verbunden ist, so dass eine Trennung unmöglich ist.

Diagnose: Tuberculose der beiden Adnexe.

Mikroskopischer Befund: Linke Tube: Mässige Auflagerungen auf der Oberfläche. Die musculäre Schicht stark verdickt und von zahlreichen Leukocyten durchsetzt. Die Schleimhaut ist vollständig bis auf einzelne Drüsenreste zu Grunde gegangen. Das Tubenlumen ist von Käsemasse ausgefüllt. Tuberkelknötchen nnd Riesenzellen sind in der musculären Schicht wenige, viele in Mucosa vorhanden.

Das linke Ovarium enthält nur noch Bindegewebsreste und ist überall von käsiger Masse, zahlreichen Tuberkelknötchen und Riesenzellen durchsetzt. An einzelnen Stellen freies Blut.

Rechte Tube: Mässige Verwachsungen auf der Oberfäche. Die musculäre Schicht ist wenig verdickt, besitzt aber zahlreiche dickwandige Gefässe und Leukocytenherde. Die Schleimhaut ist vorhanden, aber die Zotten sind untereinander verwachsen und bilden Cysten, welche von seröser Flüssigkeit angefüllt sind. Epithel ist ganz zu Grunde gegangen. In der noch vorhandenen Schleimhaut befinden sich einige Tuberkelknötchen und Riesenzellen.

Rechtes Orarium: Auf der Oberfiäche einzelne Verwachsungen; das Keimepithel ist theilweise gut erhalten. Im Rindengewebe Primärfollikel; im Ovarialstroma zahlreiche Gefässe, viele Corpora albicantia.

Diagnose: Salpingitis sinistra tuberculosa (käsige Form), Salpingitis dextra tuberculosa (miliare Form), Oophoritis sinistra tuberculosa (käsige Form), Perioophoritis dextra tuberculosa.

Am 11. [. 1909 die Patientin entlassen. In der Mitte der Bauchnarbe bestehen noch 2 Fisteln von Federkielstärke, aus denen sich eine reichliche Menge rötblich-braunen übelriechenden Eiters entleert mit leicht fäcalem Geruch.

Fall 10. Anamnese fehlt.

Operation: Laparotomie am 14. XII. 1908. Rechte Adnexe und linke Tube. Rechts hühnereigrosses Packet, bestehend aus Tube und Ovarium mit theilweise zerfetzten Wänden. Tube roth, bis fingerdick, $6 \mathrm{~cm}$ lang, im Bogen um das Ovarium ziehend, mit zahlreichen Verwachsungen an der Oberfläche. Fimbrienkranz nicht vorhanden, Lumen verschlossen, Consistenz derb. Wand glasig, sehr verdickt, Lumen eng, entleert anf Druck weissen Inhalt. Orarium zerfetzt, an der vorderen Wand mit vielen Verwachsungen. Gewebe roth, feucht mit zahlreichen kleinen Cysten durchsetzt. Am abdominalen Pol sitzt eine wallnuss- 
grosse Cyste, mit dicker, gewulsteter Innenfläche, die gelblich-eitrig anssieht und derb ist. Inhalt ist ausgeflossen.

Linke Tube $4 \mathrm{~cm}$ lang, schwillt vom uterinen bis abdominalen Ende bis zur Fingerdicke an, ist mit zahlreichen. Verwachsungen bedeckt, sehr geröthet. Fimbrienkranz nicht vorhanden. Lumen geschlossen. Consistenz fest.

Diagnose: Rechtsseitige Salpingitis. Vereitertes Corpus luteum.

Mikroskopischer Befund: Rechte Tube: Auf der Oberfläche zahlreiche Verwachsungen mit vielen Gefässen und Leukocytenherden. Die musculäre Schicht ist wenig verdickt, aber mit vielen Lymphspalten und Leukocytenherden durchsetzt. Die Schleimhaut ist vollständig zu Grunde gegangen. Das Tubenlumen ist mit käsigen Massen ausgefüllt, die aus Tuberkelknötchen und Riesenzellen bestehen.

Rechtes Ovarium: Rindengewebe locker und von Leukocyten durchsetzt. Das Ovarialstroma setzt sich zusammen aus käsigen Massen, 'Tuberkelknötchen, Riesenzellen und freiem Blut.

Linke Tube: Viele Verwachsungen auf der Oberfläche. Die Musculatur von zahlreichen Lenkocyten infiltrirt. Tubenlumen erweitert und von Käsemasse und Blut ausgefüllt. Es finden sich noch Schleimhautreste, in welchen Tuberkelknötchen, Riesenzellen und starke Leukocyteninfiltrationen vorhanden sind.

Diagnose: Salpingitis sinistra et dextra tuberculosa (käsige Form). Oophoritis dextra tuberculosa (käsige Form).

Fall 11. No. 293. 17. VII. 1909. N. N., 20jährige Glasmachersfrau, verheirathet seit 2 Jahren. Eltern, 2 Geschwister gesund, 3 Geschwister im frühen Kindesalter an Kinderkrankheiten gestorben.

Ueberstandene Krankheiten: Masern, als junges lï̈dchen bleichsüchtig. Erste Menstruation mit 14 Jahren, alle 4 Wochen regelmässig, 7-8 Tage stark; die letzten 4 oder 5 Monate weniger stark.

Die letzte Regel 22. VI. Nullipara. Schmerzen im Abdomen seit einem Jahre und Sausen in den Ohren. Schmerzen im linken Kiefergelenk. Appetit gut; Verstopfung. Harn jetzt normal (vor einem Jahr Brennen beim Harnlassen durch 8 Tage).

Die Frau ist gracil gebaut, schwächlich, blass, anämisch. Herz in normalen Grenzen, reine Herztöne; rein vesiculäres Athmen. Leichter Belag der Zunge. Bauchdecken straff. Nieren an normaler Stelle. Rechts Absturz vorhanden, weit verfolgbar. Links wölbt sich 2 Finger über Poupart'schem Bande eine ungefähr hühnereigrosse; wenig verschiebliche Resistenz vor. Brüste ohne Secretion. Vulva eng. Vagina eng und glatt, Secretion reichlich. Portio klein und kegelförmig; Uterus anteflectirt. Links von der Uteruskante ein nur schwer zu tastender hühnereigrosser Tumor, der nur wenig verschieblich ist. In den Vaginalund Urethralsecreten keine pathologischen Elemente.

Diagnose: Kystoma ovarii sinistri. Laparotomie am 22. VII. 09. Linke Adnexe und rechte Tube. Die linke Tube ist kreisförmig gebogen und hat die Form von zwei miteinander verwachsenen grossen Kartoffeln. Die Oberfläche hat zahlreiche Verwachsungen, zwischen denen viele kirschkerngrosse Knötchen zerstreut sich finden. Die Consistenz ist prall-elastisch. Reste des Fimbrienkranzes sind vorhanden; das Lumen ist geschlossen. Das linke Ovarium ist apfelgross mit zahlreichen Verwachsungen, weich-elastisch. Die rechte Tube ist $11 \mathrm{~cm}$ lang, am uterinen Ende bleistiftdick, geschlängelt und erweitert sich gegen das ab- 
dominale Ende wie ein geblähter Darm, das abdominale Ende ist geschlossen. Fimbrienkranz nicht vorhanden. Consistenz ist prall-elastisch. Die Oberfläche hat zahlreiche Verwachsungen und viele kirschengrosse Knötchen.

Diagnose: Doppelseitiger tuberculöser Pyosalpinx.

Mikroskopischer Befund: Linke Tube: Auf der Oberfläche wenig Adhäsionen mit Tuberkeiknötchen und Riesenzellen. Die Schleimhaut ist platt gedrückt, zum Theil sind noch Epithelien und Drüsenreste erhalten von Rundzellen und Tuberkelknötchen mit Riesenzellen durchsetzt. Das Tubenlumen ist von käsiger Masse ausgefüllt.

Linkes Ovarium: Auf der Oberfläche mässige Verwachsungen; an einzelnen Stellen noch Keimepithel vorhanden. In der Rindensubstanz degenerirte Primordialfollikel. Das Ovarialstroma ist locker; es finden sich noch Elemente von einem frischen Corpus luteum. Die rechte Tube zeigt denselben Charakter wie die linke Tube; auf der Serosa sind Tuberkelknötchen mit Riesenzellen vorhanden.

Diagnose: Salpingitis sinistra et dextra tuberculosa (käsige Form). Perioophoritis sinistra tuberculosa.

Fall 12. No. 107. 10. III. 1910. N. N., 47 Jahre alt, Hausmeistersfrau, verheirathet seit 17 Jahren. Vater als Bergmann verunglückt, Mutter gestorben, hatte in letzterer Zeit fortwährend Blutungen. 2 Kinder in frühester Jugend gestorben.

Die Frau war immer gesund. Erste Regel mit 15 Jahren, stets regelmässig. Letzte vor 14 Tagen. Die letzten 5-6 Perioden sind stärker als die vorhergehenden gewesen, 8 Tage statt 4, dabei übelriechender Ausfluss. Periode stets schmerzlos. Geburten: Zwei, die erste spontan, die zweite mit Zange. Scheidendammriss wurde genäht. Letzte Geburt um 1888. Keine Fehlgeburt. Die Frau giebt an Schmerzen im Unterleib zu haben seit 14 Tagen (seit der letzten Periode) meist in der linken Seite. Appetit sehr schwaeh in der letzten Zeit. Stuhl: Durchfall in den letzten Tagen. Harn o. B. Starke Blässe und Mattigkeit, bei schwachem Ernährungszustande. Lymphdrüsen o. B. Herzgrenzen nicht erweitert, Töne rein. Keine Lungendämpfung. vesiculäre Athmung. Hypogastrische und hypochondrische Gegend ganz frei. Auch bei stärkerem Druck in beiden Reg. hypogast. keine Schmerzen. Deatliche Resistenz links fühlbar. Brüste schlaff, hängend.

Portio in der Mittellinie, nach vorne gerichtet. Von der Portio setzt sich nach allen Seiten eine Geschwulst fort, die nach links bis zur Beckenschaufel geht und sich nicht von ihr abheben lässt. Dagegen geht sie rechts nicht ganz bis zur Beckenschaufel hin und ist bier von oben nach unten etwas beweglich. Auch den Douglas füllt sie ganz aus. Befund per rectum: Man stösst an der vorderen Wand an einen derben Tumor, anscheinend ein stark retroflectirter Uterus. Die Schleimhaut ist ganz beweglich. Das Lumen ist eingeengt. Urin saser, kein Eiweiss und Zucker.

Mikroskopiseher Befund des Vaginal- und Urethralsecrets: Stäbchen und grampositive Kokken; keine Gonuokokken.

Diagnose: Carcinoma uteri.

Laparotomie am 16.3. 1910. Linke Adnexe von Hübnereigrösse. Ovarium und Tube verklebt. Tube roth, sehr blutreich. Auf dem Schnitt ist der hühnereigrosse Tumor stark erweitert mit geronnenen Blutmassen ausgefüllt. Das Ovarium ist gelblich verfärbt, derb. 
Mikroskopischer Befund: Linke Tube: Auf der Oberfläche viele blutreiche Verwachsungen. Die musculäre Schicht ist dick, von Rundzellen und freiem Blut durchsetzt.

Die Schleimhaut ist vollständig zerstört und besteht aus Rundzellen und käsigen Massen. Viele Riesenzellen sind vorhanden.

Linkes Ovarium: Auf der Oberfläche blutige Auflagerungen. Im Rindengewebe befinden sich noch degenerirte Primordialfollikel. Im Ovarialstroma zahlreiche Gefässe mit verdickten Waudungen; mässige Rundzelleninfiltration. Im Ovarialstroma sind Corpora albicantia vorhanden.

Diagnose: Salpingitis sinistra tuberculosa (käsige Form). Perioophoritis sinistra tuberculosa.

In den vorstehend erwähnten Fällen scheint sich der tuberculöse Process bei der grössten Zahl der Patientinnen im Alter von 20 bis 37 Jahren gezeigt zu haben; 2 von ihnen wurden im 46. bis 47. Jahre davon ergriffen, während das Alter einer anderen nicht bekannt ist. Es offenbarte sich also die Adnextuberculose bei allen, mit Ausnahme von 3, in der Zeit des Sexuallebens. Diese Lebensperiode der Frauen hat nicht wenig Einfluss auf die Entwickelung der Tuberculose in den Geschlechtsorganen, besonders wenn sich schon in anderen Orgaven (Lungen) ein tuberculöser Process vorfindet oder wenn sich im Allgemeinen eine angeborene oder angenommene Prädisposition zur Tuberculose zeigt. Sieben Patientinnen hatten das dritte Jahr der Ehe noch nicht überschritten, als sie auch schon an den tuberculösen Adnexen operirt werden mussten. Besonders im 4. Falle gab sich die Genitaltuberculose schon nach dem 6. Monat der Ehe durch tuberculösen Ascites kund. Bei der Untersuchung fand sich Katarrh der Lungenspitzen, der schon früher bestanden und den Process auf das Peritoneum und auf die Adnexe übertragen hatte.

Wie in diesen, so begegnet man auch in fast allen anderen Fällen der tuberculösen Affection der Adnexe bei primärer Tuberculose der Lungen. Thatsächlich wurde in den Fällen 4, 5 und 7 bei der Untersuchung der Brust Lungenphthise nachgewiesen.

Der Fall, bei welchem die Patientin, kurz bevor sie sicb einer Operation unterzog, von einer rechtsseitigen Pleuritis befallen wurde, legt den Gedanken nahe, dass die Tuberculoseerkrankung der Pleura von der voraufgegangenen Infection der Genitalorgane erfolgt ist. Fernet hat derartige Fälle mitgetheilt und Hegar konnte Beweise bringen, dass die Infection auf dem Wege durch die Lymphgefässe stattgefunden hat, nachdem er gesehen hatte, dass sie mit einer käsigen Masse inficirt waren. Da jedoch in der 
Anamnese unserer Patientin keine sicheren Angaben vorhanden waren, um eine primäre tuberculöse Affection der Adnexe nachweisen zu können, lässt sich vielmehr bestimmt vermuthen, dass die Pleura durch Bacillen übersät worden ist, die von tuberculösen Herden der benachbarten Lungen herrühren. Nach meiner Meinung würde dieser Herd der Ursprung des tuberculösen Processes sowohl in der Pleura als auch in den Adnexen gewesen sein. Ebenso verhält es sich mit den anderen Fällen, mit Vorbehalt des zweiten; auch bei ihnen wird die Adnextuberculose einen secundären Ursprung gehabt haben. Es ist nicht immer leicht, wie von den Forschern allgemein angenommen wird, einen kleinen tuberculösen Herd der Lungen oder Tuberculose der peribronchialen Drüsen zu entdecken.

Während die Fälle 6 und 12 bei der Untersuchung von tuberculösen Lungenaffectionen frei waren, enthielten sie dagegen bei der Autopsie Verkäsungen der peribronchialen Drüsen. Nach den Literaturberichten citirte Bertrix gerade einen diesen beiden ähnlichen Fall und konnte bestimmt versichern, dass der Ursprung der Tuberculose der Geschlechtsorgane sich fast immer in vorher existirenden tuberculösen Affectionen der Lungen nachweisen lässt.

Der Fall 2 lässt betreffs der tuberculösen Adnexerkrankung eine von allen anderen abweichende Interpretation zu. Nachdem sich die Patientin, die ursprünglich von gesunder Constitution war, mit einem an Lungentuberculose leidenden Manne verheirathet hatte, wurde sie in den ersten Jahren ihrer Ehe von tuberculöser Adnexerkrankung befallen.

Obgleich man vermuthen konnte, dass die Infection der Adnexe durch das Sperma oder durch eine Verletzung in den weiblichen Geschlechtsorganen und somit auf dem hämatogenen Wege erfolgt sei, ist nicht auszuschliessen, dass die Patientin vom Ehemann auf dem Athmungswege tuberculös angesteckt worden sein könnte. Auf Grund dieser zweiten Hypothese, nach welcher sich der Process zuerst in den Lungen entwickelte, würde er sich dann den Adnexen mitgetheilt haben und so wäre auf solche Weise auch in diesem Falle nachgewiesen, dass die Adnexaffection secundären Ursprungs sein würde.

Die Unfruchtbarkeit, der schon von verschiedenen Forschern bei tuberculösen Frauen gedacht wurde, hat sich auch in den meisten der von mir geschilderten Fälle mit grösster Deutlichkeit gezeigt. Unter 12 Patientinnen befanden sich 8 sterile, von den 
anderen 4 hatte eine einen Abortus im 4. Monat, eine andere eine einzige Niederkunft, eine weitere zwei Geburten gehabt, während von der letzten nichts bekannt ist. Die Hypoplasie des weiblichen Sexualapparates, die von Merletti bei tuberculösen Individuen sehr häufig vorgefunden und später von anderen Autoren bestätigt worden ist, würde bei 5 meiner Fälle klar zu Tage treten, sei es in Folge zu enger Vagina, sei es durch zu kleine oder konische Portio oder schliesslich wegen eines kleinen, übertrieben anteflectirten oder retroflectirten Uterus.

Betreffs der erblichen Belastung ist mit Ausnahme des Falles 8, bei welchem eine Schwester der Kranken an Lungentuberculose starb, nichts nachzuweisen. Bei Fall 9 aber, bei dem die Patientin Carcinom der Portio zeigte, hätte man nicht auf einen begleitenden tuberculösen Process schliessen können, wie er bei der mikroskopischen Untersuchung der entfernten Organe hervortrat.

Auch die Symptomatologie der Patientinnen bot keine besonderen Merkmale von Tuberculose der Adnexe dar. Bei allen Kranken zeigten sich als Hauptmerkmale Störungen in der Menstruation, atypische Blutverluste, Ausfluss, Schmerzen verschiedentlicher Heftigkeit in der Gegend der Adnexe, alles Symptome, denen man allgemein bei der Erkrankung der Adnexe begegnet, während andererseits die allgemeinen Symptome der Tuberculose (Schweiss, Fieber, Anorhexie u. s. w.) fast immer fehlten.

Auch die bakteriologische Untersuchung des Uterinsecretes, die in allen Fällen vorgenommen wurde, hat niemals das Vorhandensein von. Tuberkelbacillen nachweisen lassen. Daher war die Diagnose nur in dem Falle 4 möglich, bei welchem die Patientin Ascites zeigte, ferner bei Fall 8, bei welchem die krankhafte Beschaffenheit der linksseitigen $\Lambda$ dnexe, die bei einer ersten Operation entfernt worden waren, bekannt war, und im 2. Fall, bei welchem man die Diagnose auf Tuberculose vor der Operation nur in Folge der klinischen Erfahrung des Operateurs stellen konnte. Dieser konnte zu der Diagnose nur gelangen durch die Angabe der Kranken, dass sie mit einem an Lungentuberculose leidenden Mann verheirathet wäre.

Es wird von allen Gynäkologen einstimmig an'genommen, dass der Tuberkelbacillus die Eigenschaft hat, besonders die Tube zu inficiren, eine Thatsache, die nach Pozzi und anderen Autoren aus den nachfolgenden Betrachtungen erklärlich ist. Die Ansammlung der krankhaften Keime wird in der Tube hervorragend 
begünstigt durch die zahlreichen Falten und die geringe Vitalităt der Schleimhaut. Thr fehlt jene Widerstandskraft, welche die Uterinschleimhaut aufweist, die zu jeder Zeit der Regel durch die theilweise Ausstossung von den vorhandenen Keimen befreit wird, und es fehlt ihr jenes platte, dicht aufeinander geschichtete Epithel, sowie die Lebenskraft der Keime, an denen die Vagina so reich ist.

Nach Schottländer bietet der Eierstock im Gegensatz zur Tube eine gewisse Widerstandskraft gegen das Eindringen und die schädliche Entwickelung der Tuberkelbacillen. Diese inficiren meistens die Eierstockdrüse, wenn sie von der Tube ber in erstere eindringen können und weun eine prädisponirende Ursache (Trauma oder eine andere vorangegangene Infection) die organische Widerstandskraft des Eierstockes verringern. Durch seine besondere Lage wird der Eierstock, zum Unterschied von der Tube, ebenfalls sehr selten isolirt vom Tuberculoseprocess ergriffen. Die Tuberculose des Eierstocks wird fast immer von Tuberculose des Peritoneums und der Tube begleitet, dagegen findet sich oft Tuberculose der Tuben an beiden Seiten und der Eierstock ist unversehrt. Terrillon sah die Tuberculose gleichzeitig in der Tube und am Eierstock 3 mal von 6 Fällen. Lea endlich constatirte beim Studium von 8 Fällen von Tubentuberculose, dass in keinem Falle der Eierstock davon ergriffen war.

Gerade meine Fälle beweisen die häufigen tuberculösen Erkrankungen der Tube gegenüber der relativen Seltenheit beim Eierstock.

Während bei allen 12 Fällen die linke Tube und in 9 Fällen die rechte Tube erkrankt war, ist der linke Eierstock nur in 7 Fällen und der rechte in 6 davon ergriffen.

Der tuberculöse Process hatte beide Tuben in 9 Fällen und die Adnexe vollständig auf beiden Seiten in 3 Fällen befallen.

Bemerkenswerth sind die zahlreichen Verwachsungen auf der Oberfläche der tuberculösen Adnexe. Sie bilden mit dem Eierstock oder der Tube ein einziges Packet von verschiedener Form und verschiedener Grösse, wobei sie jedoch nie die eines Gänseeies übertreffen.

Die Fimbrien sind im Allgemeinen verklebt, manchmal nicht mehr in den zahlreichen Adhäsionen zu unterscheiden. Die abdominelle Mündung der Tube ist oft geschlossen. Die Länge der Tube variirt zwischen einem Minimum von $3-4 \mathrm{~cm}$ und einem 
Maximum von $9 \mathrm{~cm}$, ihr Umfang überragt im Allgemeinen den eines Fingers, nur im 11. Falle erreicht er den des Dickdarms. Meistens hat die Tube eine geschlängelte Gestalt. Selten erstrecken sich alle erwähnten pathologischen Veränderungen der Tube auf deren Uterinende, das sich mikroskopisch fast immer normal zeigt.

In einigen Fällen sind stecknadelkopfgrosse Tuberkelknötchen auf der Serosa der Tube und auf den Adhäsionsgeweben sichtbar. Das Tubenlumen ist manchmal mit einer weisslichen festen, manchmal mit einer derben glasigen Masse angefüllt, die mit der Wand eng verbunden ist, so dass eine Trennung unmöglich ist, manchmal endlich mit einer geronnenen blutigen Masse. Der mit mehr oder weniger zahlreichen Adhäsionen bedeckte Eierstock weist verschiedene Form, Consistenz und Grösse auf. Auch wenn er in einen wirklichen Abscess verwandelt ist, überschreitet er nie die Grösse eines Hühnereies.

Sein Inhalt besteht aus gelbem, dünnflüssigem Eiter und die Innenfläche dèr Abscesswandung ist von grünlich gelber Farbe, stark zerklüftet und mit eitrig fibrinösen, nicht abstreifbaren Auflagerungen bedeckt. Der rechtsseitige Eierstock des Falles 6 hatte an der vorderen, oberen Fläche eine über ein Markstück grosse Perforationsöffnung, die während der Operation entstanden war.

Fasst man die feineren mikroskopischen Veränderungen an den Tuben ins Auge, so fanden sich $1 \mathrm{mal}$ an der linken Seite (Fall 4) und $3 \mathrm{mal}$ an der rechten Seite $(5,6,9)$ folgende pathologische Merkmale.

Die Tubenoberfläche ist mit Adhäsionen bedeckt, welche grösstentheils zahlreiche neugebildete, mit Blut angefüllte Grefässe haben.

Unterhalb der Serosa finden sich manchmal eigenthümlich langgestreckte Spalträume, welche mit cubischem Epithel ausgekleidet sind. Die muskuläre Schicht der Tubenwand ist hypertrophisch mit kleinzelliger Infiltration, eigentliche Tuberkelknötchen findet man sehr selten.

Die grossentheils von Rundzellen infiltrirte Schleimhaut ist mit zahlreichen, typischen Tuberkeln und Riesenzellen besetzt. Die Schleimhautfalten schwellen stark kolbig an, verkleben untereinander und verwandeln sich in Granulationsgewebe. Durch gegenseitige Verklebung bilden sie oft Cysten, die mit seröser Flüssigkeit angefüllt sind. 
Das Oberflächenepithel ist nicht immer verloren. In der Tiefe der Schleimhaut bleiben manchmal abgeschnürte Epithelreste erhalten.

Die verkäste Tuberculose findet sich in unseren Fällen am häufigsten und zwar 5 mal rechts und 11 mal links. Das Tubenlumen ist mit käsiger Masse angefüllt, die Schleimhaut ist fast. gänzlich zerstört.

Die Musculatur ist oft dünn, infiltrirt von Leukocyten und besetzt von Tuberkelknötchen mit Riesenzellen. Auf der Serosa. finden sich zahlreiche Adhäsionen, die ebenfalls von Leukocyten infiltrirt sind und manchmal mit Tuberkelknötchen und Riesenzellen besetzt sind. In der rechten Tube im Falle 3 begegnet man innerhalb der Schleimhaut nur wenigen miliaren Tuberkeln; käsige Massen fehlen vollkommen; während sich in der musculären und Schleimhautschicht die Bildung von fibroiden Geweben bemerkbar macht, das sich manchmal in und zwischen die Tuberkelknötchen vertheilt.

In diesem Falle lässt sich die chronische fibroide Form nachweisen, die zuerst von Williams unterschieden wurde. Williams glaubt, dass es sich hier ursprünglich am entzündliche Veränderungen handelt, die theilweise fortbestehen, theilweise abgelaufen waren, so dass die tuberculöse Erkrankung ein durch Entzündung verändertes Gewebe befallen und so vielleicht einen besonders günstig vorbereiteten Boden gefunden hat.

Auch in den Ovarien sind sowohl die tuberculöse Perioophoritis, wie die eigentliche 0 varialtuberculose deutlich ausgeprägt.

Die erste Form (tuberculöse Perioophoritis) zeigt sich bei dem linksseitigen Eierstock der Fälle 1, 2, 3, 7, 8, 11, 12 und bei dem rechtsseitigen Eierstock des Organs vertheilt, während das Uebrige normal ist. Ein mehr oder weniger grosser Theil der Ovarialoberfläche ist mit dicken, zusammenhängenden tuberculösen Schwarten bedeckt, welche sich zum grössten Theil aus lauter confluirenden kleineren Knötchen zusammensetzen. Manchmal ist das Keimepithel unter den tuberculösen Auflagerungen streckenweise noch theils im normalen theils in etwas comprimirtem Zustande erhalten.

Der zweite Process (Ovarialtuberculose) zerfällt in drei verschiedene Formen, die miliare, die käsige und die abscedirende und tritt in folgenden Fällen und auf folgende Weise auf: Die miliare Form im rechtsseitigen Eierstock des Falles 2; sie kenn- 
zeichnet sich durch das Vorbandensein von isolirten Tuberkelknötchen mit Riesenzellen im Stroma. Die Rundzelleninfiltration ist im Stroma stark, in der Rindensubstanz mässig.

Die käsige Form findet sich im linksseitigen Eierstock der Fälle 5, 9 und im rechtsseitigen Eierstock der Fälle 3, 7, 10.

Hier bemerkt man nicht nur isolirte Tuberkelknötchen mit Riesenzellen, sondern auch grösstentheils käsige Massen und einzelne Riesenzellen im Stroma.

Die abscedirende Form im rechtsseitigen Eierstock der Fälle 5 und 6 unterscheidet sich von den anderen durch folgende Merkmale:

Das ganze Stroma und ein Theil der Rindensubstanz ist in einen Abscess verwandelt. Seine Wand besteht aus Granulationsgewebe und vielen kleinen, neugebildeten Gefässen und zahlreichen polynucleären Leukocyten. In der Abscesshöhle findet man eine theils fluctuirende, theils anhaftende käsige Masse, mit Tuberkelknötchen und Riesenzellen. Zwischen der Abscesswand und der Rindensubstanz besteht eine stark infiltrirte Zone. Die Rindensubstanz ist locker und mässig infiltrirt. Auf der Oberfläche des Eierstockes sind wenig Verwachsungen.

Beim linken Eierstock des Falles 6 begegnet man keinerlei Spuren von Tubereulose, jedoch stellt er das Bild einer chronischen interstitiellen Oophoritis dar.

Sonach ergiebt sich, dass die Tube häufiger tuberculös ist als das Ovarium. Dies bekräftigt die von allen Forschern bereits angenommene Idee, dass die Widerstandskraft des Eierstockes grösser ist, als die der Tube. Während die Tuben fast immer auf beiden Seiten durch den tuberculösen Process ernstlich erkrankt. sind, zeigen sich die Ovarien dagegen oft vollständig gesund oder mit einem auf ihrer Peripherie localisirten Process. Die Häufigkeit und Form des tuberculösen Processes in den Adnexen aller 12 Fälle geht aus folgender Tabelle hervor.

Wenden wir uns nun zur Diagnose der Adnextuberculose, so verursacht sie in fast allen Fällen grosse Schwierigkeiten. Sie war nur in den wenigen Fällen möglich, bei welchen das Vorhandensein von Ascites und die allgemeine Symptomatologie der Patientin unmittelbar darauf hinwies. (Im Fall 2 die Verheirathung mit einem an schwerer Lungentuberculose erkrankten Manne.)

Bei den meisten Fällen war die Diagnose nur durch den mikroskopischen Befund und durch die mikroskopische Untersuchung der 
340 Loiacono, Beitrag zur Tuberculose der weiblichen Adnexe.

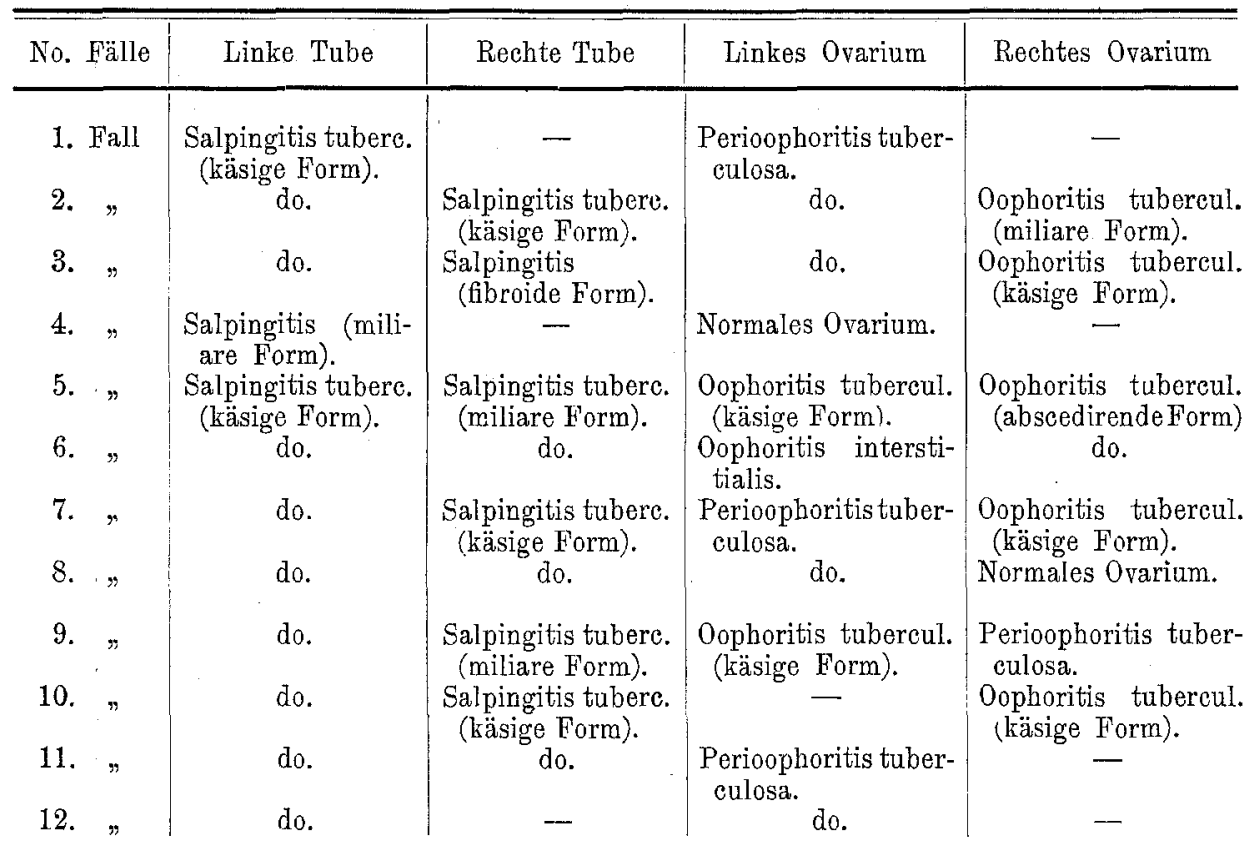

entfernten Adnexe zu stellen. Ich muss deshalb ebenso wie die anderen Autoren zugeben, dass zur Zeit die Diagnose der Adnextuberculose meistens schwierig, wenn nicht oft sogar unmöglich ist.

Die in den letzten Jahren auch in der Gynäkologie geübte Tuberculinreaction hat bisher noch keine genügenden Resultate erzielt.

Die Prognose der Adnextuberculose ist sehr ernst: 3 von den 12 Patientinnen sind sofort nach der Operation gestorben.

Die Ursache liegt in der häufigen Zusammenwirkung des Tuberculoseprocesses der Lungen mit dem der Adnexe. Andererseits kommen oft die Patientinnen erst sehr spät zum Gynäkologen, weil die tückische Ausbreitung der Tuberculose und das häufige Fehlen ernster Schmerzen die Kranke nicht rechtzeitig auf den Process, der ihre Existenz bedroht, aufmerksam machen.

Der 2. und 3. Fall, in welchem die Patientinnen zur Zeit in guter Gesundheit waren, bekräftigen diese Ansicht. Der 2. Fall in Sonderheit verdankt seinen guten Ausgang nicht nur dem rechtzeitigen Eingriff des Operateurs, sondern auch dem Umstand, dass die Adnextuberculose mit grosser Wahrscheinlichkeit nur in diesen Organen localisirt war. Nachdem sie von dem Herde befreit war, erlangte sie die volle Gesundheit wieder. 
Die Therapie endlich war in allen 12 Fällen eine chirurgische, und zwar wurde stets der abdominelle Weg befolgt. Bei 8 Fällen wurden auf beiden Seiten die Adnexe entfernt, bei 3 Fällen nur an der linken Seite operirt, in 1 Falle verblieb der rechtsseitige Eierstock.

Hieraus geht hervor, dass der Operateur eine conservirende Absicht, namentlich den Ovarien gegenüber, befolgt hat.

Anders liegt die Sache bei den Tuben. Diese müssen nicht nur gründlich entfernt werden, weil die Tuberculose darin einen gutèn Boden zur Entwickelung findet, sondern auch deshalb, weil der Gynäkologe darnach trachten muss, bei tuberculösen Individuen oder solchen, die zur Tuberculose prädisponirt sind, so zu handeln, dass sie nicht der Gefahr einer Schwangerschaft ausgesetzt werden können.

Dagegen empfiehlt es sich, mit der Entfernung der Eierstöcke vorsichtig zu sein, auch wenn dieses Organ während der Operation leicht pathologisch erscheint, da durch die Befreinng des Organismus von den tuberculösen Herden in den Tuben das Ovarium mit grosser Wahrscheinlichkeit seinen vollkommen normalen Zustand wieder annehmen kann.

Auf dem Congress in Rom erklärten Veit und Truzzi, bei Operationen von peritonealer Tuberculose gute Resultate mit der Ausspülung der Bauchhöhle mittelst leichter Formalinlösungen erzielt zu haben. Eine solche Behandlung könnte man auf die tuberculösen Adnexe vielleicht ausdehnen. Wenn diese Ausspülung auch keine grosse desinficirende Wirkung hätte, so könnte sie doch wenigstens die operirten und benachbarten Gegenden von tuberculösen Keimen, die während der operativen Eingriffe eventuell dort verblieben sind, mechanisch befreien.

\section{Literaturverzeichniss.}

Amann, Cong. périod. internat. de Gynécologie et Obstétrique. Comptes Rendus. 4 Sess. Rom Sept. 1902.

Baisch, Die Dauerresultate bei der Behandlung der Peritoneal- und Genitaltuberculose. Verhandl. d. Deutschen Gesellsch. f. Gyn. XII.

Birnbaum, Die Erkennung und Behandlung der Urogenitaltuberculose mit dem Koch'schen Tuberculinpräparate. Verhandl. d. Deutsehen Gesellsch. f. Gyn. Dresden.

Blau, Ueber die Entstehung und Verbreitung der Tuberculose im weiblichen Genitale. Münch. med. Wochensehr. 1909. No. 31. 
Faure, Congrés pér. intern. de Gyn. et Obst. Rom Sept. 1902.

Glimm, Beitrag zur Aetiologie der Tuberculose. Inaug.-Dissert. Greifswald, Hegar, Die Entstehung, Diagnose und chirurgische Behandlung der Genitaltaberculose. Stuttgart 1886, Deutsche med. Wochenschr. 1897. No. 45. Münch. med. Wochenschr. 1900.

$\mathrm{Hoh} / \mathbf{f} \in \mathbf{l d}$, Tuberculose der inneren weiblichen Genitalien im Kindesalter. Sitzungsber. d. Gesellsch. f. Gyn. zu Leipzig. Febr. 1907.

$\mathrm{Klob}$, Pathologische Anatomie der weiblichen Sexualorgane. S. 372.

Koblank, Clinica contemporanea.

Jung and Bennecke, Experimentelle Untersuchungen über Infectionswege bei der weiblichen Genitaltubereulose. Dieses Archiv. Bd. LXXX.

Josselin de Jong, Fall von Carcinom und Tuberculosis uteri. Ref. Centralblatt f. Gyn. 1907.

Lea, Remarks on tubercular disease of the fallopian tube with analysis of eight cases. Journ. of obst. a. gyn. of Brit. Empire. April 1905.

Martin, Congrés pér. intern. de Gyn. et Obst. Rom Sept. 1902. - Zur Genitaltuberculose. Berl. klin. Wochenschr. 1908. No. 3. - Krankbeiten der Eierstöcke.

Macnaugton, Jones, Ueber primäre unilaterale Tuberculose der Tuba Follopii. Royal soc. of med. of London. Gyn. sect. April 9.

Merletti, citirt von Amann.

Pankow, Verhandl. der Deutschen Gesellsch. f. Gyn. XII.

Picherin, Congrés pér. intern. de Gyn. et Obst. Rom 1902. - Semaine Gynécologique. 1909.

Pozzi, Congrés pér. intern. de Gyn. et Obst. Rom 1902. - Traité de Gynécologie.

Spinelli, Congrés pér. intern. de Gyn. et Obst. Rom 1902.

Trutzi, Ibidem. Rom 1902

Terillon, Archives de tocologie. 1889.

Veit, Handbuch der Gynäkologie. 'III. Bd. - Congrés pér. intern, de Gyn. et Obst. Rom 1902.

Williams, J. W., Tuberculosis of the female generative organs. John Hopkins Hospital Report. Baltimore 1892. 